Chapter 9

\title{
Polarization State Manipulation of Electromagnetic Waves with Metamaterials and Its Applications in Nanophotonics
}

\author{
Shuqi Chen, Wenwei Liu, Zhancheng Li, \\ Hua Cheng and Jianguo Tian \\ Additional information is available at the end of the chapter
}

http://dx.doi.org/10.5772/66036

\begin{abstract}
Polarization state is an important characteristic of electromagnetic waves. The arbitrary control of the polarization state of such wave has attracted great interest in the scientific community because of the wide range of modern optical applications that such control can afford. Recent advances in metamaterials provide an alternative method of realizing arbitrary manipulation of polarization state of electromagnetic waves in nanoscale via ultrathin, miniaturized, and easily integrable designs. In this chapter, we give a review of recent developments on polarization state manipulation of electromagnetic waves in metamaterials and discuss their applications in nanophotonics, such as polarization converter, wavefront controller, information coding, and so on.
\end{abstract}

Keywords: polarization manipulation, optical activity, wave plates, wavefront controlling, optical communication

\section{Introduction}

Harnessing electromagnetic waves for modern nanophotonics applications often involves the control and manipulation of polarization state. The ability to manipulate the polarization state of electromagnetic waves can enable us to control electromagnetic waves for a wide range of applications such as polarization manipulation, wavefront controlling, and optical communication [1-4]. Conventional approaches to manipulate the polarization state of electromagnetic waves employ bulky wave-plates, which are made of birefringent materials composed of crystalline solids and liquid crystals. However, the inherent disadvantages in 
the size, collimation, and bandwidth of these configurations prevent optical system miniaturization and integration. Thus, realizing polarization state manipulation of electromagnetic waves in nanoscale has become one of the key problems for the development of modern optics and nanophotonics.

Metamaterials are generally composed of subwavelength artificial nanostructures, which can overcome the physical limitations imposed by natural materials and provide exceptional capabilities for manipulating waves with greater precision. Over the past decade, numerous novel optical properties have been demonstrated in this area, such as negative refractive index, super-lenses, cloaking, etc. [5-14]. The soul of metamaterials is the ability to realizing arbitrary manipulation of the electromagnetic waves in multiple parameters (frequency, amplitude, phase, and polarization) with multiple degrees of freedom, which make it possible for people to design devices with optical properties on demand. Recently, metamaterials have been reported to provide a promising pathway toward the realizing of efficient manipulation of polarization state of electromagnetic waves via ultrathin, miniaturized, and easily integrable designs, which open up intriguing possibilities toward the realization of polarization state manipulation of electromagnetic waves in nanoscale and show infinite prospection in nanophotonics applications [4, 5, 15-17].

In this chapter, we will review the development of metamaterials in polarization state manipulation of electromagnetic waves and discuss its applications in nanophotonics, which will provide a guidance for its further designs and applications. The chapter is organized as follows:

In the second section, we begin with a brief introduction of polarization state of electromagnetic waves and give a review of the development process of metamaterials for polarization state manipulation.

In the third section, the fundamental applications of metamaterials in nanophotonics, such as wave plates, polarization converter, opt-isolator, arbitrary generation of vector beam, polarization-based wavefront-control, and so on will be discussed in detail.

In the fourth section, the polarization integrated metamaterials devices, such as tunable polarization controlling devices, photonic spin Hall effect, information coding and optical communication, and polarization-switchable phase holograms, will be discussed in detail.

In the fifth section, we will overlook the whole area of metamaterials-based polarization devices, summarize the main difficulties, possible solutions, and further applications in future.

In the last section, we will give a summary of the chapter.

\section{Polarization state manipulation in metamaterials}

\subsection{Polarization state of electromagnetic waves}

The polarization state of electromagnetic waves, which cannot be detected by human eyes, forms an important characteristic of such waves. The polarization state of electromagnetic waves at a fix position is determined by the time course of the electric-field vector $\mathbf{E}(\mathbf{r}, t)[18]$. 
For monochromatic electromagnetic waves, the electric-field vector $\mathbf{E}(\mathbf{r}, t)$ can be divided into two orthogonal components with different amplitude and phases, which can be concisely expressed in the form of Jones vector as:

$$
\mathbf{E}(\mathbf{r}, \mathbf{t})=\left(\begin{array}{c}
\mathbf{E}_{x} \\
\mathbf{E}_{y}
\end{array}\right)=\left(\begin{array}{c}
a_{x} e^{i\left(k x-\omega t+\varphi_{x}\right)} \\
a_{y} e^{i\left(k x-\omega t+\varphi_{y}\right)}
\end{array}\right)
$$

The endpoint of the vector $\mathbf{E}(\mathbf{r}, t)$ is determined by the envelope of two orthogonal components $A_{x}=\operatorname{Re}\left(\mathbf{E}_{x}\right)$ and $A_{y}=\operatorname{Re}\left(\mathbf{E}_{y}\right)$, which vary in cosinusoid with time and the trajectory of the endpoint can be expressed as:

$$
\frac{A_{x}^{2}}{a_{x}^{2}}+\frac{A_{y}^{2}}{a_{y}^{2}}-2 \cos \varphi \frac{A_{x} A_{y}}{a_{x} a_{y}}=\sin ^{2} \varphi
$$

where $\varphi=\varphi_{y}-\varphi_{x}$ is the phase difference. The trajectory of Eq. (2) is an ellipse and the properties of it vary with the position for wavefront with different directions at different positions. For the plane wave whose wavefront is parallel transverse plane, the elliptical trajectory stays the same. Thus, the polarization state of plane wave can be described by a single ellipse. Moreover, such polarization ellipse can be determined by its orientation and shape which can be characterized by two angles:

$$
\begin{aligned}
& \tan 2 \Psi=\frac{2 r}{1-r^{2}} \cos \varphi, \\
& \sin 2 \chi=\frac{2 r}{1+r^{2}} \sin \varphi .
\end{aligned}
$$

where $\mathrm{r}=a_{x} / a_{y}$ is the ratio of the magnitude of two orthogonal electric field components. The angle $\Psi$ determines the orientation of the polarization ellipse while the angle $\chi$ determines the ellipticity. Thus, the polarization state of electromagnetic waves can be described by the magnitude ratio $r=a_{x} / a_{y}$ and phase difference $\varphi=\varphi_{y}-\varphi_{x}$. For $a_{x}$ (or $\left.a_{y}\right)$ equal to zero or the phase difference $\varphi=0$ (or $\varphi=\pi$ ), the polarization ellipse becomes a straight line, and the electromagnetic waves is linearly polarized (LP). If the magnitude ratio is $r=1$ and the phase difference is $\varphi= \pm \pi / 2$, then the polarization ellipse become a circle and the electromagnetic waves is said to be circularly polarized. For $\varphi=\pi / 2$ (electric field rotates in a clockwise direction when viewed from the direction toward which the wave is propagating), the electromagnetic waves is said to be right-handed circularly polarized (RCP). And the case $\varphi=-\pi / 2$ corresponds to counterclockwise rotation and left-handed circularly polarized (LCP). For other condition, the polarization state of electromagnetic waves is said to be elliptically polarized.

\subsection{Polarization state manipulation with metamaterials}

As indicated above, the polarization state of electromagnetic waves can be described by magnitude ratio $r$ and phase difference $\varphi$. Thus, the polarization state manipulation with metamaterials always involves the tailoring of the wave interference at the subwavelength scale by 
introducing the anisotropic optical resonance mode to effectively manipulate the magnitude and phase of electric components in two orthogonal directions. Thus, the polarization state manipulation of electromagnetic waves in metamaterials always involves structures with two orthogonal resonance including elliptical nanoholes, L-shaped nanoparticles, crossed nanodipoles, nanoslits, and nanorods, as shown in Figure 1 [19-24].

However, the polarization state manipulative ability of initial complanate metamaterials with the above-mentioned structures is limited because of the limited interaction between electromagnetic waves and structures, thus the efficiency and bandwidth are lower than that required for practical applications. Over the past decade, many efforts have been made in the scientific community to overcome the drawbacks of complanate metamaterials and improve the polarization state manipulation of electromagnetic waves in nanoscale with new types of metamaterials $[25,26]$. As one of the solutions, sandwich-like metamaterials constructed with anisotropic resonators, dielectric layer, and highly reflective metallic film have been proposed to provide an alternate way to realize effective polarization state manipulation in reflection mode as shown in Figure $\mathbf{2 a}$ and $\mathbf{b}$ [27, 28]. The near-field interference in this type of designs improves the interaction between electromagnetic waves and metamaterials effectively, thus realizing
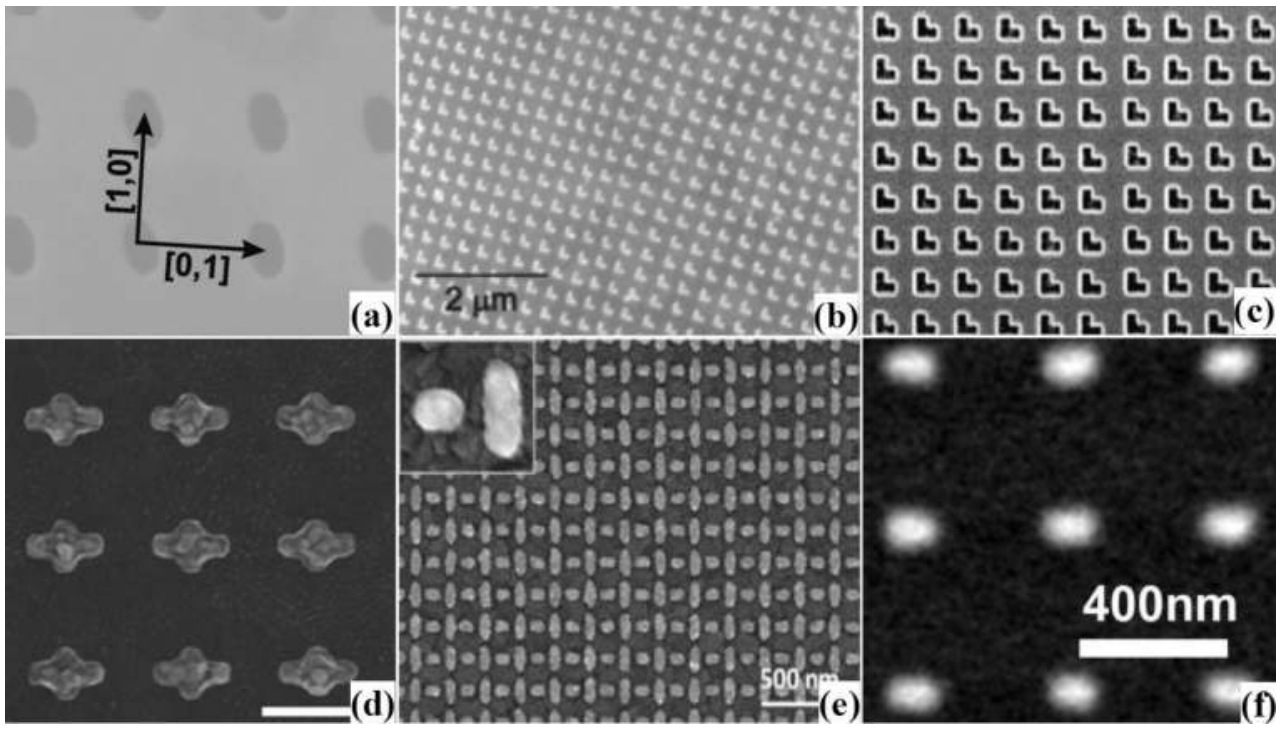

Figure 1. (a) Experimental realization of strong polarization controlling in the optical transmission through elliptical nanoholes. Reprinted by permission from [19] copyright 2004 American Physical Society. (b) Experimental realization of birefringence in two-dimensional L-shaped silver nanoparticles. Reprinted by permission from [20] copyright 2008 American Chemical Society. (c) Experimental realization of manipulating the polarization state of the electromagnetic waves and achieving giant optical rotation in near infrared wavelength by L-shaped nanoholes array in silver film. Reprinted by permission from [21] copyright 2008, AIP Publishing LLC. (d) Experimental realization of chromatic plasmonic polarizers by optical nanorods. Reprinted by permission from [22] copyright 2012 American Chemical Society. (e) Experimental realization of broadband optical meta-waveplates by crossed nanodipoles. Reprinted by permission from [23] copyright 2013 American Chemical Society. (f) Experimental realization of reflecting wave plates constructed with nanodipoles. Reprinted by permission from [24] copyright 2014 Optical Society of America. 
effective polarization state manipulation of electromagnetic waves in reflection mode. On the other hand, applications in modern nanophotonics always require nanoscale polarization state manipulation in transmission mode. Thus, sandwich-like metamaterials is out of work in this situation. Recently, the proposition of few-layer metamaterials makes it possible to realize effective polarization state manipulation in transmission mode as shown in Figure $2 \mathbf{c}$ and $\mathbf{d}$ [29, 30]. The interference and near-field coupling between layers in few-layer metamaterials ensure that the energy of electromagnetic waves can be strongly redistributed and can effectively interact with the structures, resulting in polarization state manipulation with high efficiency and broadband in transmission mode. However, the above-mentioned metamaterials are all based on metallic structures, and thus the electromagnetic waves absorption and subsequent heat conversion in sandwich-like and few-layer metamaterials inevitably increase which impeded the applications of metallic structure-based metamaterials for polarization state manipulation

(a)

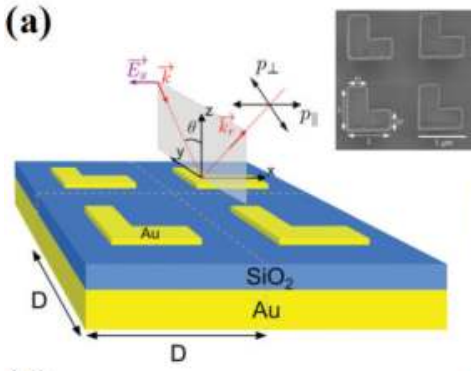

(d)

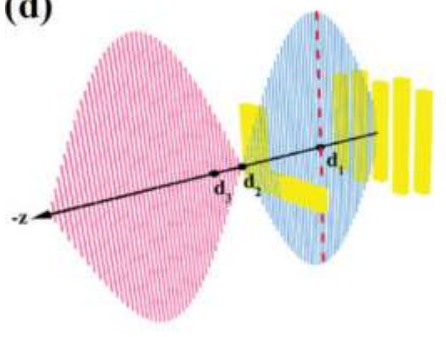

(b)

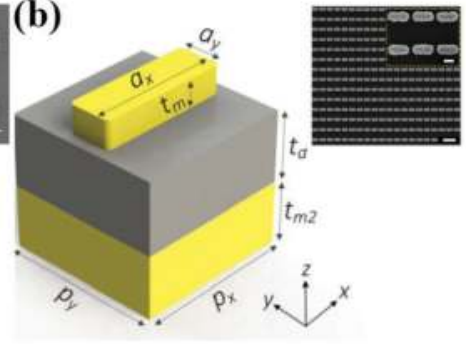

(c)

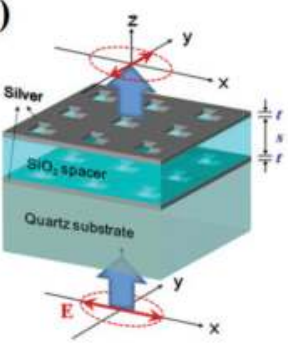

(e)

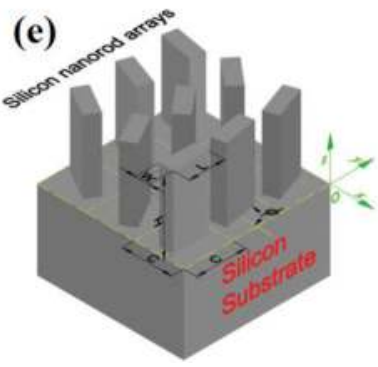

(f)

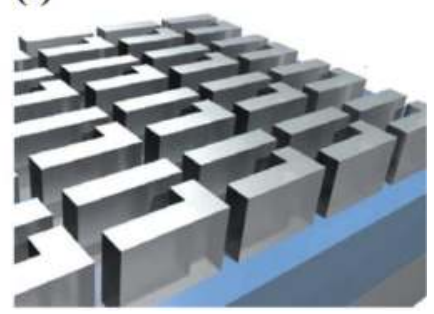

Figure 2. (a)Experimental realization of wide band and efficient linear polarization conversion with plasmonic planar antenna. Reprinted by permission from [27] copyright 2014 AIP Publishing LLC. (b) Experimental realization of wide-angle reflective plasmonic metasurface-based half-wave and quarter-wave plates that have a high polarization conversion efficiency and reflection magnitude over a broad bandwidth in the visible-to-near IR wavelength range. Reprinted by permission from Macmillan Publishers Ltd [28], Scientific reports copyright 2016. (c) Experimental realization of a plasmonic assisted Fabry-Perot cavity in a metal/insulator/metal trilayer structure with L-shaped hole arrays inside, which significantly contribute to the mechanism to realize a nearly complete polarization conversion in optical transmissions at near-infrared wavelength. Reprinted by permission from [29] copyright 2010, AIP Publishing LLC. (d) Simulated realization of a high performance broadband asymmetric polarization conversion composed of an L-shaped gold particle and a gold nanoantenna array for the near-infrared regime. Reprinted by permission from [30] copyright 2015 Springer International Publishing AG. (e) Simulated realization of all-silicon nanorod-based Dammann gratings with accurate phase controlling, and strong polarization conversion. Reprinted by permission from [31] copyright 2015 Optical Society of America. (f) Experimental realization of silicon-based infrared metasurfaces as high performance linear-to-circular polarization converter. Reprinted with permission from Macmillan Publishers Ltd, [32] Nature Communications copyright 2014. 
of electromagnetic waves. Recently, a different approach has emerged. The Mie resonance in high-index dielectric structures provides a novel way to realize anisotropic optical resonance [31, 32]. Metamaterials based on all-dielectric nanoparticles (as shown in Figure $2 \mathbf{e}$ and $\mathbf{f}$ ) overcome the energy loss of electromagnetic waves in metallic structure-based metamaterials and become an ideal selection for polarization state manipulation of electromagnetic waves.

In summary, metamaterials with the development of it can realize the effective controlling of amplitude and phase of electromagnetic waves in two orthogonal directions, which provide infinite possibilities for arbitrary manipulation of polarization state of electromagnetic waves in nanoscale. Thus, the role of metamaterials for polarization manipulation devices in nanophotonics is no substitute. With the improvement of the relative research, metamaterial-based polarization manipulation devices have been widely proposed in recent years which will be discussed in detail in the next section.

\section{Fundamental applications of metamaterials for polarization state manipulation in nanophotonics}

As mentioned above, polarization state is one of the intrinsic properties of electromagnetic waves, which can always be resolved into different orthogonal basis, such as $x$-polarized and $y$-polarized, left-handed and right-handed waves. It is worth mentioning that the leftright-handed polarization corresponds to the spin of photons, which enables metamaterials with great abilities of polarization conversion to be a possible quantum candidate in large scale. In modern optics and photonic applications, polarization conversion is often utilized in advanced communication, sensing, displayer, noise reduction, and so on. However, most of these applications come down to three categories: conversion of polarization and generation of vector beams, wave plates, and asymmetric transmission. In this section, we aim at providing an overall view on the applications and evaluating the possibilities of commercial utilization of metamaterials.

\subsection{Conversion of polarization and generation of vector beams}

Metamaterials have shown to the world their unique design flexibility, compactness, and highly novel characteristics at its very first birth. Unlike the traditional method, in which polarization is often controlled with polarized molecules (such as liquid crystals) [33], birefringent crystals [18], or magneto-optic phenomenon (Faraday Effect) [34], metamaterials can directly manipulate strength and phase of electromagnetic waves at subwavelength scale. In the eyes of a metamaterials' researcher, polarization of electromagnetic waves can be modified pixel by pixel (unit cell of the design), thus arbitrarily polarized outputs on Poincare Sphere can be easily achieved [35].

Throughout the recent works, there are two kinds of metamaterials polarization converters that have shown their powerful ability to handle polarization. One is as shown in Figure 3a, which is an enhanced optical rotator of the zero-order transmitted electromagnetic waves through a silver film with an array of perforated S-shaped holes [36]. The fundamental mechanisms of 
these kinds of polarization rotators are as follows: the incident wave interacts with the subwavelength structure and creates orthogonal polarized component due to the surface plasmon polaritons (SPPs), or localized surface plasmons (LSPs), or both. With elaborate design, the original polarized electric component can be cancelled due to near-field interference, and the orthogonal one is left over, thus a cross-polarization converter is achieved. Other states of polarization can also be accomplished with similar method because the complete orthogonal basis has been acquired. The transmitted polarization depends on the thickness of the device, as shown in Figure $\mathbf{3 b}$, which exhibits a tiny ellipticity, indicating the transmission can be roughly treated as a linearly polarized one [21, 31, 37].

Recently another design almost dominates the area of polarization conversion with metamaterials, as shown in Figure 3(c) to 3(f). This kind of designs simultaneously manipulate the amplitude and phase of electric-magnetic components [35], [38]-[41], resulting in much higher degree of freedom for polarization conversion. In Figure 3(c), the incident linearly polarized (LP) wave can be converted into left-circularly polarized (LCP) and right-circularly polarized (RCP) beams at sub-wavelength scale [38]. The difference of phase retardation between the LCP and RCP beams can be easily modified by varying the geometrical parameters of the nano-apertures, leading to continuously controllable optical activity. It's worth mentioning that most designs generate orthogonal electric components by the chiral-
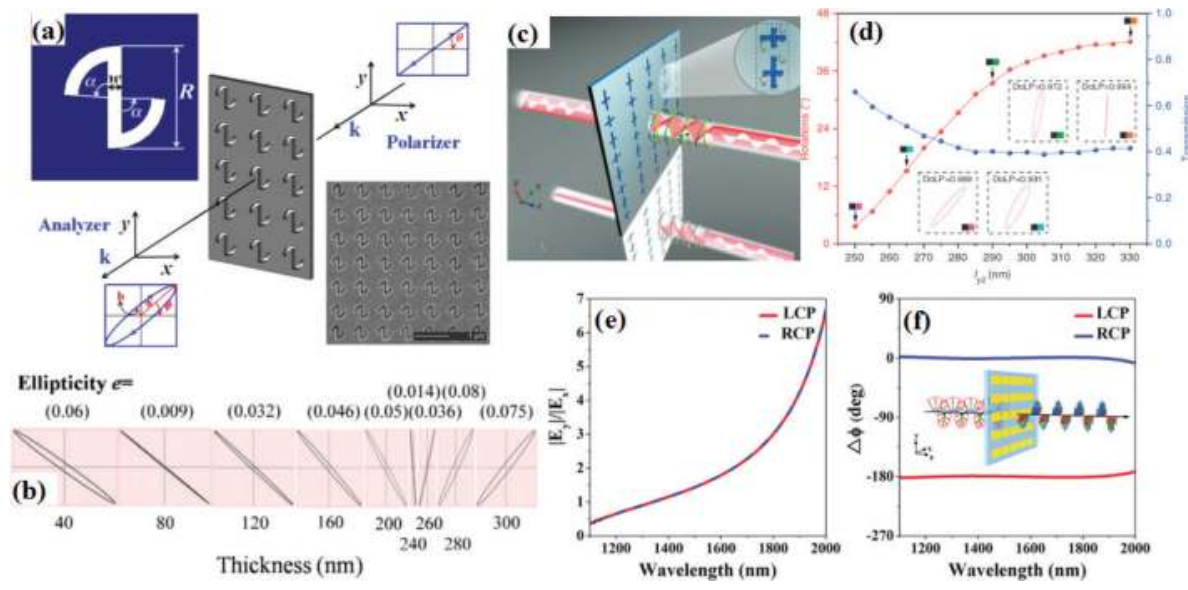

Figure 3. (a) Schematic of the experimental set up for the transmitted polarization converter, in which the sample made from silver is typically built with focused ion beam (FIB) method. In the experiment, the incident wave remains linearly polarized along the $x$ axis. (b) The transmitted elliptical polarization states and the corresponding ellipticities for different samples with different thicknesses. Reprinted by permission from [36] copyright 2013 American Physical Society. (c) Sketch of the optical activity process with non-chiral plasmonic metasurfaces, in which the transmitted linearly polarized wave is synthesized with two subwavelength located circularly polarized wave. (d) Calculated optical rotations (red lines) and amplitude transmissions (blue lines) at a wavelength of $990 \mathrm{~nm}$. The insets show the polarization states and the corresponding degree of linear polarization (DoLP) for four metasurfaces. Reprinted by permission from Macmillan Publishers Ltd [38], Light: Science and Applications, copyright 2016. (e) Simulated amplitude ratio and (f) phase difference of the electric components of the transmitted waves for right- and left-circularly polarized incidence, respectively. Inset: artistic rendering of the circular-to-linear (CTL) polarization converter. Reprinted by permission from Macmillan Publishers Ltd [39], Scientific reports, copyright 2015. 

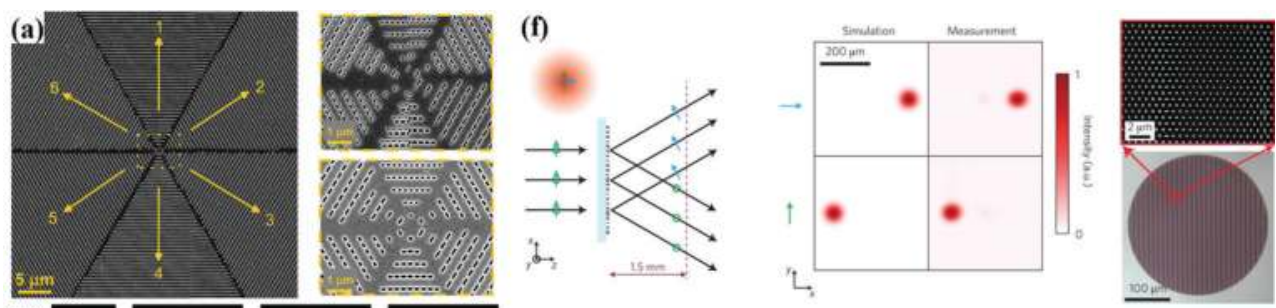
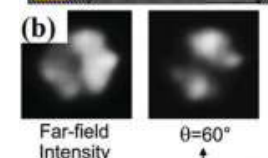

$\theta=60^{\circ}$
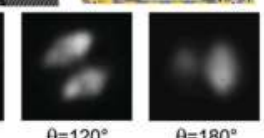

$\theta=180^{\circ}$

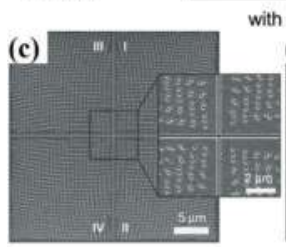

with polarizer

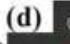

(d) $0 \quad 0$

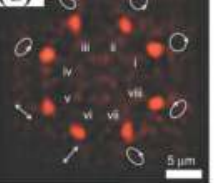

(e)
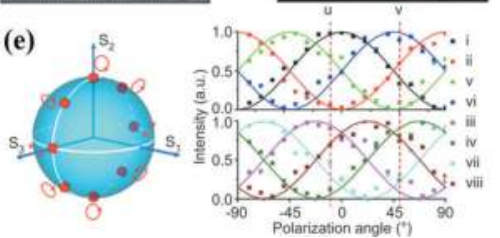

(g)

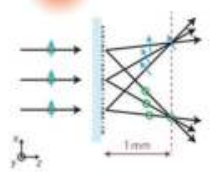

(h)
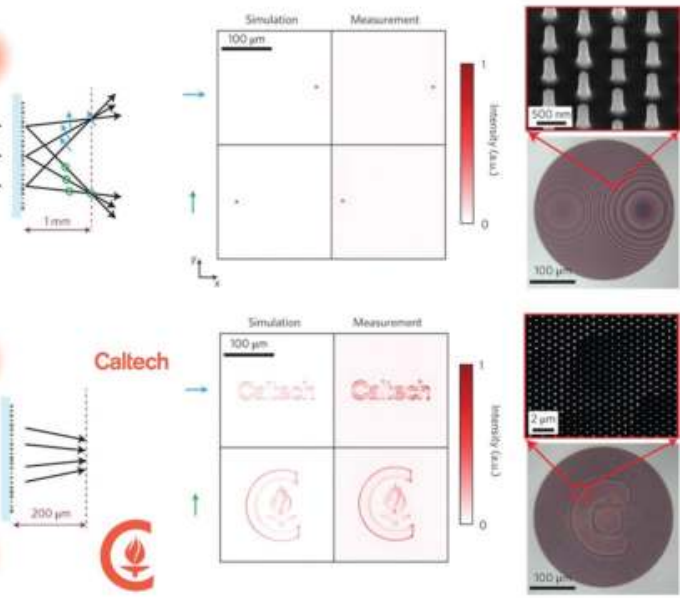
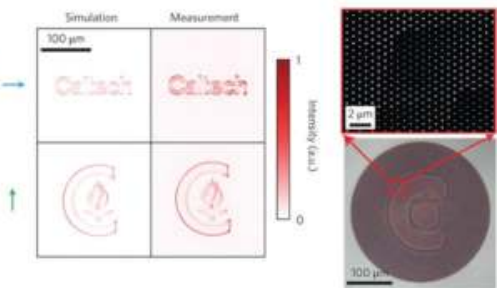

Figure 4. (a) SEM image of the plasmonic metasurface to generate a radially polarized beam. Each region of the plasmonic metasurface is filled with one type of nano-aperture pairs marked by the number. (b) Measured far-field intensity profiles of the radially polarized beam, with an analyzer oriented at various angles in front of the CCD camera. Reprinted by permission from [14] copyright 2015 by John Wiley and Sons. (c) SEM image of the polarization generator for the eight-foci process. (d) Focus image without polarization analysis, each hot spot of which represents one of the polarization states of the foci. (e) The polarization states of the foci on the Poincare sphere, and Si stands for the ith Stoke's parameter. Reprinted by permission from Macmillan Publishers Ltd [42], Light: Science and Applications, copyright 2015. (f) A polarization beam splitter that separate the $x$ - and $y$-polarized waves and deflects them by different angles, and the angles can be chosen at will. (g) A device that separates $x$ - and $y$-polarized waves and focuses them to two different points. The two different points can be chosen at will. (h) A polarization-switchable phase hologram that generates two arbitrary patterns for $x$ - and $y$-polarized waves. In this case the word "Caltech" is displayed for input $x$ polarization and an icon is displayed for input $y$ polarization. Reprinted by permission from Macmillan Publishers Ltd [35], Nature nanotechnology copyright 2015.

ity of the materials or nano-structures, however the design in Figure 3(c) experimentally and numerically investigated an alternative approach to realize and control the optical activity with non-chiral plasmonic metasurfaces. The optical rotation was calculated in Figure 3(d), and rotation with arbitrary values from $3^{\circ}$ to $42^{\circ}$ is obtained, with relatively high-amplitude transmission beyond $40 \%$. Circularly to linearly polarized wave can also be achieved in [39], as shown in Figure 3(e) and 3(f). The inset in Figure 3(f) depicts the artistic rendering of the design. As amplitude ratio varies throughout the working waveband, indicating different orientation of transmitted wave when differing the incident wavelength, this device can hardly called a broadband quarter-wave plate. However, it is still a novel polarization converter, and may be utilized in a metasurface displayer to distinguish different colors. Since this design is a 
time reversal symmetric system (taking no account of the thermal loss), it can also be applied to convert linearly polarized wave to circularly polarized one.

As mentioned above, the subwavelength characteristics of metamaterials enable people to modify polarization of electromagnetic waves pixel by pixel (unit cell of the design), and to generate wave with different polarization in each cross-section of the outputs. As shown in Figure $4 \mathbf{a}$, a radially polarized beam is generated by appropriately arranging the nano-apertures [14]. Although the six regions are discontinuous with each other, the achieved vector beam is continuous due to the subwavelength characteristics of the structure, which is always a principle of the metamaterials' design. A far-field intensity profile of the radially polarized beam is shown in Figure $4 \mathrm{~b}$. Furthermore, with the in-plane field of SPPs combined, all types of polarization states can be achieved simultaneously [42], as shown in Figure $4 \mathbf{c}-\mathbf{e}$. This remarkable consequence results from that the in-plane field of SPPs with proper polarization states and phases can be selectively scattered out to the desired electromagnetic wave beams. This design offers a novel route to achieve the full control of optical polarizations. However, all these devices are plasmonic based and the efficiency is limited by the intrinsic absorption loss. Although many researchers struggled to overcome this limitation by introducing multilayered structures or performing at reflectance mode [24,27], and so on, it can hardly be solved thoroughly due to the high conductivity of metals. In recent years, high-contrast dielectric designs have been developed to enlarge the working efficiency and practicability of metamaterials [26]. As shown in Figure $\mathbf{4 f - h}$, full control of polarization for beam splitter, lens, and phase hologram is achieved with a simple layer of silicon elliptical nanoposts [35], with an experimentally measured efficiency ranging from 72 to $97 \%$. The complete and simultaneous control over the polarization and phase profiles of electromagnetic waves offered by the proposed platform and the design technique enables the realization of integrated nanooptic devices, which is one of the greatest steps in modern optics and photonics.

\subsection{Wave plates accomplished by metamaterials}

Although full control of polarization is the primary issue with respect to polarization, wave plates are still worth discussing due to their wide applications in applied optics. Normally wave plates are manufactured from birefringent crystals, which are really successful due to their high efficiency and accuracy. However, simultaneously achieving broadband and wideangle properties are almost impossible because of the limitation of the crystals' dispersive properties. In contrast, metamaterials once again provide a promising pathway toward the perfect wave plates with thickness less than a micrometer. As shown in Figure 5a, a broadband half-wave plate is accomplished by a nanorod layer and a metallic reflective ground plane sandwiched by a dielectric layer [28]. An s-polarized wave incident from an angle of $\theta_{i}\left(\varphi_{i}=135^{\circ}\right)$ is converted into a $p$-polarized wave in reflectance mode, with reflection greater than $92 \%$ from 640 to $1290 \mathrm{~nm}$, and with near unitary polarization conversion ratio (PCR) even when increasing the incident angle to $40^{\circ}$. This phenomenon results from the asymmetric length of the long and short sides of the nanorod, which responds to the incident beams with different phase delay and radiative intensity. Similarly, a quarter-wave plate can also be obtained with careful adjustment of dimensions of the structure. As shown in Figure $\mathbf{5 c}$, the calculated results are in good agreement with the experimental ones. Although the designed 

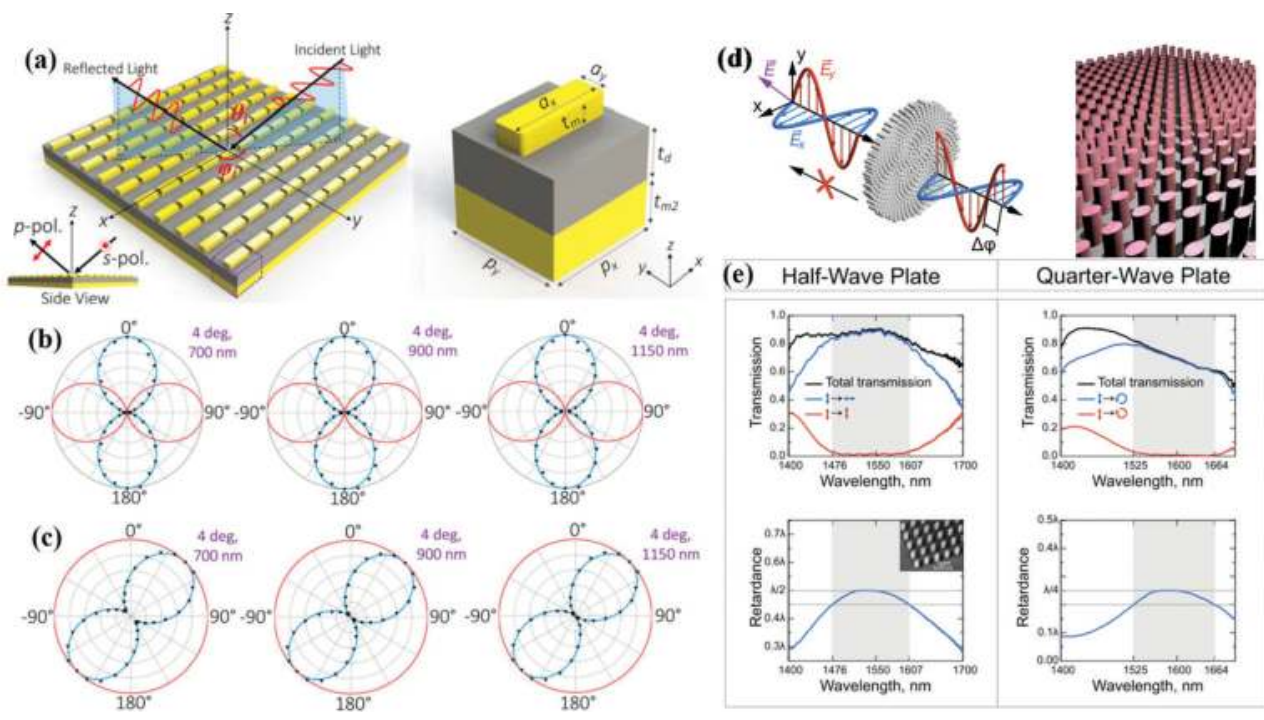

Figure 5. (a) Schematic of the metasurface-based half-wave plate. An s-polarized wave incident from an angle of $\theta_{i}\left(\varphi_{i}=\right.$ $135^{\circ}$ ) is converted into a $p$-polarized wave in reflectance mode. The unit cell shows the sandwiched metallic structure. (b) Theoretically predicted (lines) and experimentally measured (triangles) polarization states in the plane perpendicular to the wave vector at 700, 900, and $1150 \mathrm{~nm}$ incidence, respectively. (c) Theoretically predicted (lines) and experimentally measured (triangles) polarization states in the plane perpendicular to the wave vector at 700, 900, and 1150 nm incidence, respectively, for the design of quarter-wave plate. Reprinted by permission from Macmillan Publishers Ltd [28], Scientific reports copyright 2016. (d) An artistic view of a meta-device for spatially variant polarization control operating in a broad spectral range. The device is composed by a single layer of elliptical dielectric nanoposts. (e) High efficient half-wave plate and quarter-wave plate achieved by the dielectric nanoposts. Reprinted by permission from [43].

wave plates are broadband and wide-angled, especially with high efficiency, a critical drawback still exists that they work in reflectance mode, compared to which transmission mode is often preferable because of its intrinsic convenience for experimental and commercial utilization. One way to solve this problem is to use high-contrast dielectric metasurfaces [43], which possess the ability to interact with electromagnetic waves at extremely confined spots without any heat dissipation, as depicted in Figure 5d and e. The designed half-wave plate and quarter-wave plate maintain a high transmission in the working waveband, and the undesired component of polarization is suppressed at a negligible level.

\subsection{Asymmetric transmission}

Another application on polarization is asymmetric transmission, which are often utilized in integrated photonic systems for communications and information processing. This unique phenomenon is often achieved by reducing the structural symmetry and converting to different polarization states. The corresponding process can be completely characterized by the $2 \times 2$ ray-transfer matrix in the paraxial ray-optics approximation. We can write 
down the $T$ matrix connecting the generally complex amplitudes of the incident and the transmitted field:

$$
\left(\begin{array}{c}
E_{x}^{o} \\
E_{y}^{o}
\end{array}\right)=\left(\begin{array}{ll}
T_{x x} & T_{x y} \\
T_{y x} & T_{y y}
\end{array}\right)\left(\begin{array}{c}
E_{x}^{i} \\
E_{y}^{i}
\end{array}\right)=\left(\begin{array}{cc}
A & B \\
C & D
\end{array}\right)\left(\begin{array}{c}
E_{x}^{i} \\
E_{y}^{i}
\end{array}\right)
$$

where ' $o$ ' and ' $i$ ' denote the incident and output field, respectively. If the structure is rotated by $180^{\circ}$ with respect to the $x$ axis (assuming the wave vector is along $z$ axis), it can be demonstrated that the T matrix (backward) can be written as [44]:

$$
\mathbf{T}^{b}=\left(\begin{array}{cc}
A & -C \\
-B & D
\end{array}\right)
$$

Similarly for circularly polarized incidence the $\mathbf{T}$ matrices are:

$$
\mathbf{T}_{\text {circ }}^{f}=\left(\begin{array}{cc}
T_{++} & T_{+-} \\
T_{-+} & T_{-}
\end{array}\right)
$$

and

$$
\mathbf{T}_{\text {circ }}^{b}=\left(\begin{array}{cc}
T_{++} & -T_{-+} \\
-T_{+-} & T_{--}
\end{array}\right)
$$

where the linearly and circularly polarized matrices are related by

$$
\left(\begin{array}{ll}
T_{++} & T_{+} \\
T_{+} & T_{-}
\end{array}\right)=\frac{1}{2}\left(\begin{array}{ll}
A+D+i(B-C) & A-D-i(B+C) \\
A-D+i(B+C) & A+D-i(B-C)
\end{array}\right)
$$

The difference between the $\mathbf{T}$ matrices for opposite propagating direction is the reason for asymmetric transmission, which can be defined as $\Delta=\left|T_{11}^{f}\right|^{2}+\left|T_{21}^{f}\right|^{2}-\left|T_{11}^{b}\right|^{2}-\left|T_{21}^{b}\right|^{2}$. For linear polarization $\Delta=|C|^{2}-|B|^{2}$, and for circular polarization $\Delta=\left|T_{+}\right|^{2}-\left|T_{+-}\right|^{2}$.

One of the designed metasurfaces with asymmetric transmission is shown in Figure 6a and b, which demonstrates a highly efficient and broadband asymmetric transmission of linearly polarized millimeter waves [45]. The remarkable consequence results from the tri-layer configuration, which can be promoted to other designs of metasurfaces and this will be discussed in detail in Section 5. At optical waveband, asymmetric transmission can also be achieved, as shown in Figure 6c. The fabricated devices designed for operation at central wavelength of 532 and $633 \mathrm{~nm}$, exhibit broadband, efficient asymmetric optical transmission with contrast ratios exceeding $14 \mathrm{~dB}$ [46]. The FDTD-simulated amplitude of the magnetic field at an arbitrary time is shown in Figure $\mathbf{6 d}$. It is clear to see that with forward incidence (from Side A to $B)$, the transmitted field displays a profile of diffraction, and with backward incidence (from Side B to A), the transmitted field is blocked. As stated previously, the primary way of achieving asymmetric transmission is by reducing the structural symmetry and converting 

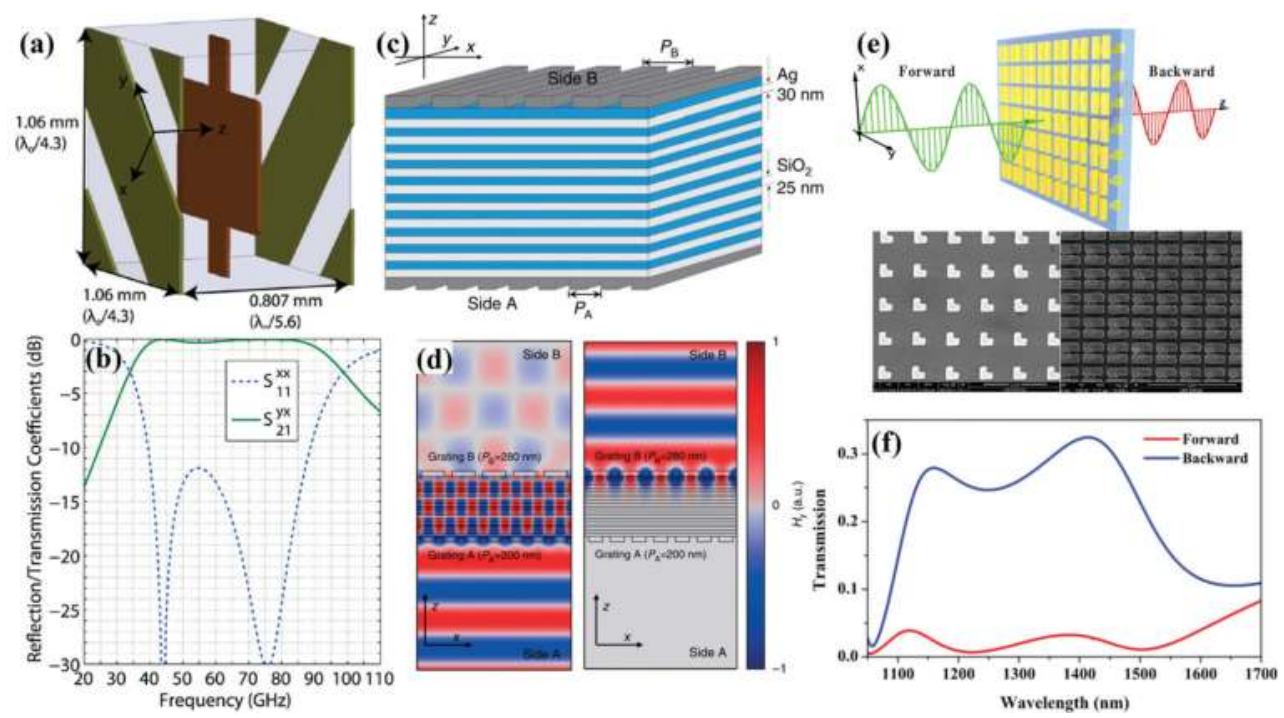

Figure 6. (a) Schematic of the tri-layer metasurface which enables (b) broadband and highly asymmetric transmission of linearly polarized waves. Reprinted by permission from [45] copyright 2014 American Physical Society. (c) Schematic diagram of the $\mathrm{Ag} / \mathrm{SiO}_{2}$ hyperbolic metamaterial device, which exhibits asymmetric transmission shown in (d). Electromagnetic waves illuminate from side A to side B on the left graph in (d) and side B to A on the right one. Reprinted by permission from Macmillan Publishers Ltd [46], Nature communications copyright 2014. (e) Asymmetric transmission achieved by a bi-layer metasurface consisting of an L-shaped layer and a nanorod layer. (f) Transmission of the $x$-polarized waves from forward and backward, respectively. Reprinted by permission from [47] copyright 2014 AIP Publishing LLC.

between polarization states, thus the metasurfaces can actually be more concise, as shown in Figure 6e. The hybrid metasurface consists of an L-shaped metallic layer and a nanorod layer. With $x$-polarized incidence, the forward and backward transmission reveals a great difference between 1200 and $1500 \mathrm{~nm}$ [47], the simulated results of which are illustrated in Figure 6f. It should be noticed that asymmetric transmission is different from optical isolator, which can only be attained with nonreciprocal-active devices.

In summary, full control of polarization can now be obtained with metallic or high-contrast dielectric-based metamaterials, the remarkable abilities of which can be attributed to the indepth subwavelength design of metamaterials. Practical applications, such as vector beams, wave plates, and asymmetric transmission devices, can be accomplished with high efficiency, and other particular designs such as broadband or wide-angle one can also be acquired at will. Other novel applications of polarization integrated devices will be discussed in the next section.

\section{Polarization integrated metamaterials devices}

As discussed in the previous section, metamaterials has made remarkable development in nanophotonics devices for polarization state manipulation of electromagnetic waves. With the design of individual unit cell of metamaterials instead of the entire structure, metamateri- 
als can not only manipulate the amplitude and phase of the electromagnetic waves but also realize the controlling of the wavefront of electromagnetic waves, thus it provides an ideal way to get the optical devices on demand and shows great impact on nanophotonics. These days, with the ever-increasing demand for integrated optical devices, the research direction is shifting toward achieving tunable, integrated, and novel functionalities.

\subsection{Tunable metamaterials devices}

Electromagnetic waves controlling in nanophotonics integration always require the devices with the tunability of waveband and functionalities. The polarization state manipulation of electromagnetic waves in earlier metamaterials for different working wavebands is always realized by accurately fabricating different nanostructures, which is an inherent drawback for integration $[48,49]$. One way to overcome this drawback is to use tunable metamaterials, which relies on integrating metamaterials with optically active materials such as liquid crystals, semiconductors, phase-change material, and nonlinear media [50-54]. The optical response of these metamaterials can be actively controlled by external stimulus, such as electric field, magnetic field, voltage, or temperature. Among all these techniques, voltage control is one of the simplest ways in practical operations. Graphene is a monolayer of hexagonally arranged carbon atoms that can support the excitation of surface plasmons and its optical response shows a strong dependence on the Fermi energy, which can be dynamically controlled by a gate voltage [55-57]. Therefore, graphene is a promising electrically tunable plasmonic material. The investigation of tunable plasmons in graphene nanostructures has led to the proposition and demonstration of a variety of devices for polarization state manipulation of electromagnetic waves. Figure 3a shows a mid-IR highly tunable optical polarization converter composed of asymmetric graphene nano-crosses [58]. It can convert linearly polarized wave to circularly and elliptically polarized wave or exhibit a giant optical activity at different wavelengths. The transmitted wavelength and polarization states can also be dynamically tuned by varying the Fermi energy of graphene (as shown in Figure $\mathbf{7 b}$ and c), without reoptimizing and refabricating the nanostructures. This devices is potentially useful in applications, such as vibrational circular dichroism spectroscopy, ellipsometry, and integration of other optical devices for polarization manipulation, detection, and sensing at the nanoscale. Figure $\mathbf{7 d}$ shows a mid-IR highly wavelength-tunable broadband cross-polarization converter based on L-shaped graphene nanostructures [59]. It can convert linearly polarized wave to its cross-polarization in the reflection mode. The polarization conversion can be dynamically tuned and realize a broadband effect by varying the Fermi energy (as shown in Figure 7e and $\mathbf{f}$ ). This tunable polarizers (or polarization switchers) provide an alternate way for the waveband controlling of polarization state manipulation. A step further, not only the waveband and functionalities of polarization state manipulation can be tuned with graphene-based metamaterials, Figure $7 \mathrm{~g}$ shows tunable wavefront controlling of crosspolarized electromagnetic waves based on periodically patterned graphene nano-crosses in the infrared regime [60]. With this device, the wavefront of cross-polarized circular refraction waves can be effectively controlled with the polarization conversion induced geometric phase and the working waveband can be dynamically tuned (as shown in Figure $7 \mathbf{h}$ and $\mathbf{i}$ ). This active wavefront controlling device can be treated as polarization and spectral beam splitters at nanoscale. 

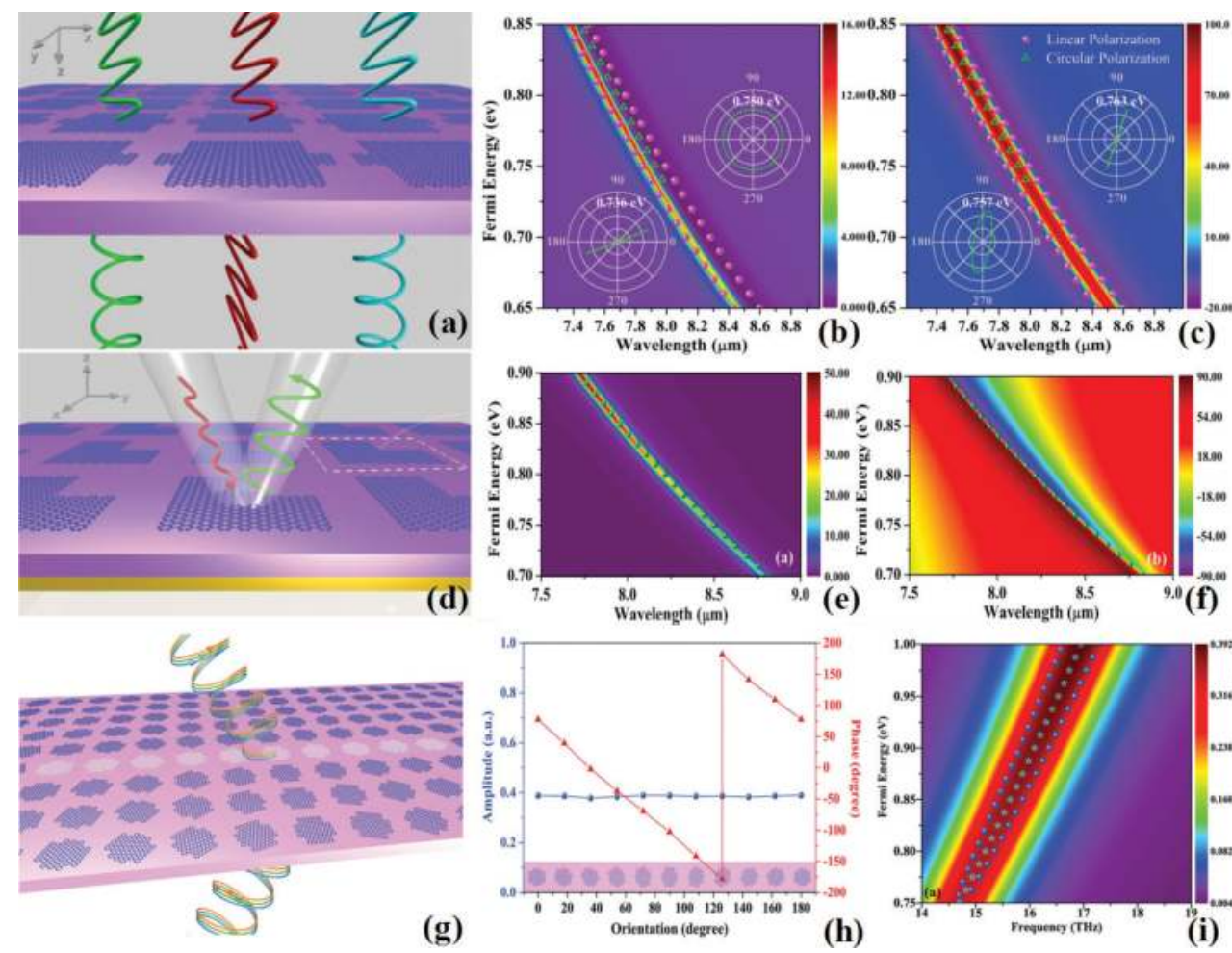

Figure 7. (a) Simulated realization of highly tunable optical polarization converter. (b) Calculated amplitude ratio and (c) phase difference as a function of the Fermi energy and wavelength. Inset images show the transmitted polarization state for different Fermi energies at a wavelength of $7.92 \mu \mathrm{m}$. The incident wave is linearly polarized with a polarization angle of $45^{\circ}$. Reprinted by permission from [58] copyright 2013 Optical Society of America. (d) Simulated realization of highly wavelength-tunable broadband cross-polarization converter. (e) Calculated amplitude ratio and (f) phase difference between two orthogonal polarization states as a function of Fermi energy and wavelength. The olive balls indicate that the reflection phase difference is $0^{\circ}$. Reprinted by permission from [59] copyright 2013 AIP Publishing LLC. (g) Simulated realization of highly tunable broadband wavefront controlling. (h) Calculated transmission amplitude and phase profile of the refraction wave along the graphene nano-cross array. (i) Amplitude of anomalous refraction of LCP wave as a function of Fermi energy and frequency, at normal incidence of RCP wave. The pentagrams and balls indicate the maximum amplitude and the extracted equal amplitude of anomalous refraction, respectively. Reprinted by permission from [60] copyright 2012 by John Wiley and Sons.

In general, tunable metamaterial devices (especially graphene-based one) provide an effect way to realize the polarization state manipulation of electromagnetic waves for nanophotonics integration because of the tunability of waveband and functionalities they have.

\subsection{Novel applications of polarization integrated metamaterials}

As mentioned above, metamaterial-based polarization converter can not only realize the transform of polarization state of electromagnetic waves, but also control the wavefront of the cross-polarized wave by inducing the geometric phase gradient via individual unit cell design. This character makes metamaterial devices become an ideal unit for integrated manipulation of electromagnetic waves, thus providing endless possibilities for novel applications in nanophotonics. 

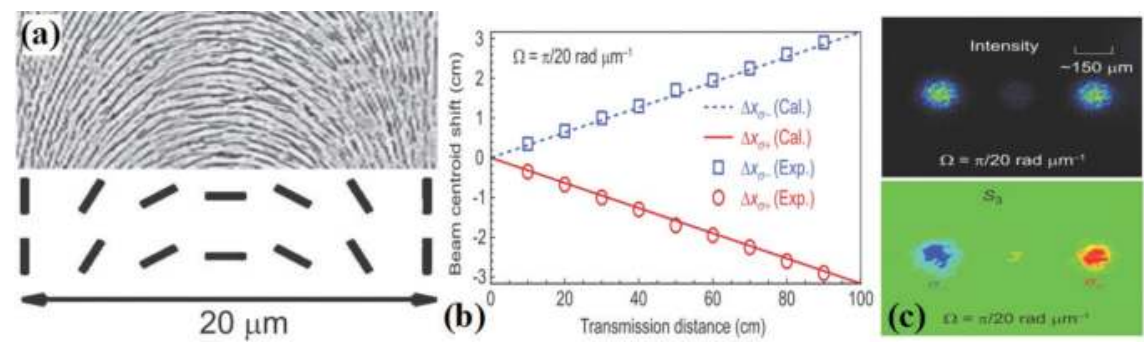

Figure 8. (a) The detailed geometry of the metamaterial over one period and a schematic illustration of the local optical axis (slow axis). (b) The calculated (Cal.) and experimental (Exp.) results of spin-dependent real-space shift induced by the momentum shift during beam with a geometric phase gradient of $\Omega=\pi / 20 \mathrm{rad} \mu \mathrm{m}-1$. (c) Intensities recorded by the $\mathrm{CCD}$ and the corresponding $\mathrm{S}_{3}$ parameters after a linearly polarized beam passes through metamaterial. The observation plane is located $10 \mathrm{~cm}$ away from the metamaterial. Reprinted by permission from Macmillan Publishers Ltd [65], Light: Science and Applications, copyright 2015.

\subsubsection{Gain spin-orbit interaction of electromagnetic waves with metamaterial devices}

Recently, spin-orbit interaction of electromagnetic waves attracted lots of attention [61]. The spin-orbit interaction of electromagnetic waves are analogous to the spin-orbit interaction of relativistic quantum particles and electrons in solids with a spatial scale of the order of the wavelength of electromagnetic waves, the RCP and LCP polarization states correspond to two spin states of photons. Thus, traditional geometrical optics always neglects the wavelengthscale spin-orbit interaction phenomena. With the development of nanophotonics and plasmonics, spin-orbit interaction phenomena play an important role at the subwavelength scales and bring novel functionalities to optical nanodevices. Photonic spin Hall effect, which manifests itself as the mutual interplay between the photon spin (polarization) and the trajectory (orbital angular momentum) of electromagnetic waves, is one of the basic classes of numerous spin-orbit interactions phenomena at the subwavelength scales. Traditional approaches to realize the photonic spin Hall effect are always associated with the evolution of the propagation direction of electromagnetic waves. However, the photonic spin Hall effect in these approaches are generally very weak, and the induced spin-dependent subwavelength shifts are also exceedingly tiny which prevent them to real applications in nanophotonics. Spindependent geometric phase gradient in metamaterials provide an alternative method to realize a gain on spin-orbit interaction at nanoscale [40, 62-64]. Figure 8a-c shows an experimental demonstration of a giant photonic spin Hall effect at a visible wavelength in a dielectric-based metamaterial device with spin-dependent geometric phase gradient [65]. The spin-dependent shift induced by geometric phase gradient is sufficiently large to be observed directly compared with traditional approaches. These kinds of devices bridge the gap between spin-based photonics and nanophotonics and thus provide an opportunity for manipulating the spin and orbital angular momentum of electromagnetic waves.

\subsubsection{Information coding and wave coding with metamaterial devices}

Modern optical communication always involves the effective device for information coding. Polarization integrated metamaterial devices provide an alternative way to realize the information coding via manipulating and detecting the polarization state of electromagnetic waves. Recently, metamaterial devices with the ability to dynamically tune the polarization state of 

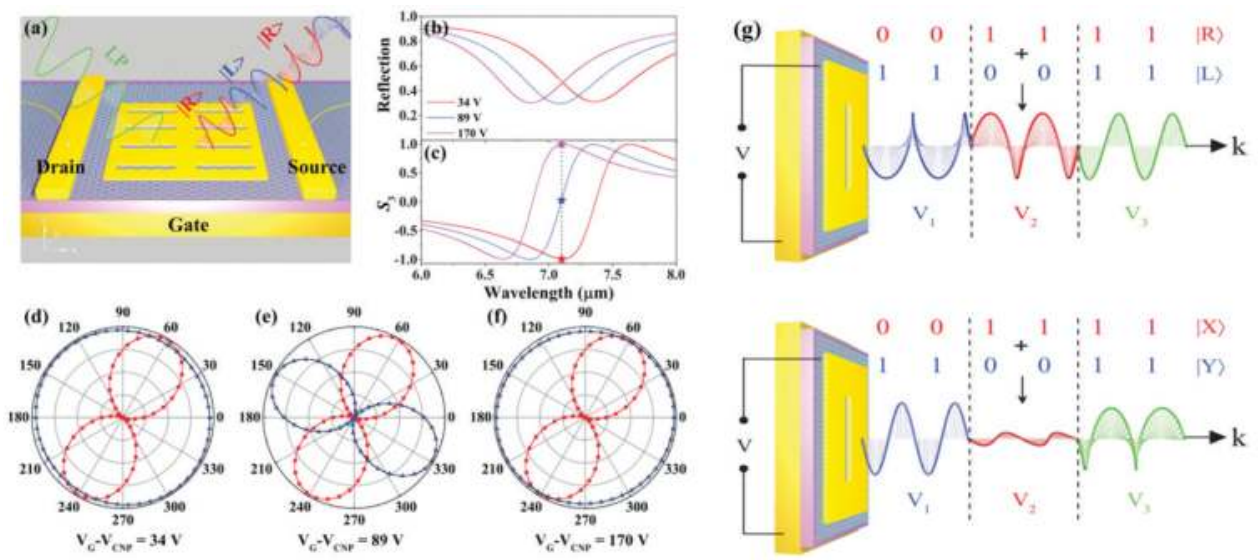

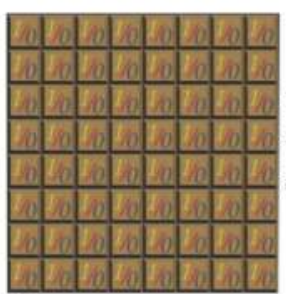

(h)

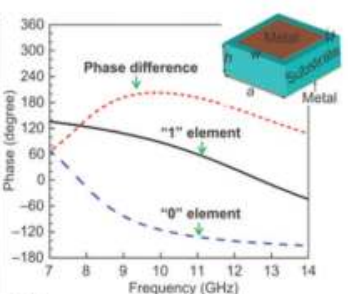

(i)

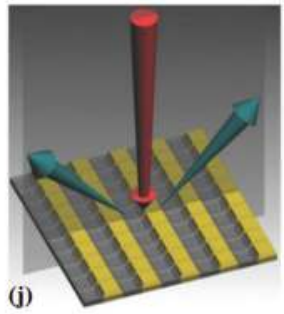

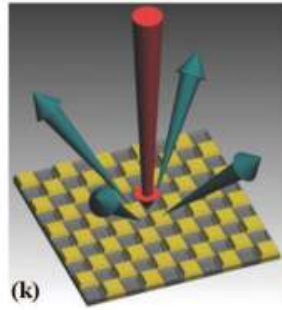

Figure 9. (a) Schematic illustration of the optical polarization encoding process based on the circular orthogonal polarization basis. (b) and (c) Simulated amplitude and normalized Stokes parameter $\mathrm{S}_{3}$ spectra of the reflected electromagnetic wave from the nano-aperture array at different gate voltages under incident wave with a polarization direction of $61^{\circ}$ relative to the $x$-axis. The stars indicate the values of $S_{3}$ under different gate voltages at $7.1 \mu \mathrm{m}$. (d)-(f) Simulated polarization state in the plane perpendicular to the wave vector at $7.1 \mu \mathrm{m}$ for different gate voltages. Red and blue curves correspond to the incident and reflected wave, respectively. (g) Schematic illustration of realizing the PDM technique by the proposed metamaterial device with the circular and linear orthogonal polarization basis according to the superposition principle of polarization states. Polarization states of electromagnetic waves correspond to three difference gate voltages. Reprinted by permission from [66] copyright 2016 by John Wiley and Sons. (h) The 1-bit digital metamaterial device composed of only two types of elements: "0" and "1." (i) A square metallic patch unit structure (inset) to realize the " 0 " and " 1 " elements and the corresponding phase responses in a range of frequencies. Two 1-bit periodic coding metasurfaces to control the scattering of beams by designing the coding sequences of " 0 " and "1" elements: (j) the $010101 \ldots / 010101 \ldots$ code and (k) 010101.../101010... code. Reprinted by permission from Macmillan Publishers Ltd [67], Light: Science and Applications, copyright 2015.

electromagnetic waves have attracted enormous interest because such devices can be employed for realizing not only the polarization encoding but also polarization-division multiplexing, which is a crucial technique that can significantly increase the transmission capacity of a single physical channel. The traditional technique for realizing polarization-division multiplexing requires a complex optical system and cumbersome volume. Therefore, a metamaterial-based polarization modulator offers a new approach for simplifying the optical process and miniaturizing the required volume. Figure 9a shows a metamaterial device by integrating a single layer of graphene with an anisotropic metamaterial, which can dynamically modulate the polarization state of electromagnetic waves with a wide tunable range in mid-infrared wavelengths 
[66]. By switching gate voltage applied on the graphene among three different values, the incident LP wave can be dynamically converted into LCP, RCP, or linearly cross-polarized one in the reflection direction, as shown in Figure $\mathbf{9 b}$-f. Based on these polarization-control characteristics, the proposed device can realize polarization encoding and the polarization-division multiplexing technique, as shown in Figure $9 \mathrm{~g}$. This design profoundly affects a wide range of modern optical communication devices, fulfills the demand of faster information transfer and processing, and opens a route to on-chip integration of metasurfaces with electronics.

Another approach named "coding metamaterial" has also been paid great attention by the scientific community, which is composed of several types of unit cells, with different constant phase responses, respectively [67-70]. Figure $9 \mathbf{h}$ shows a 1-bit coding metamaterial, composed of two types of unit cells, with 0 and $\pi$ phase responses (as shown in Figure 9i), which is named " 0 " and " 1 " elements, respectively [67]. This coding metamaterial device can simply

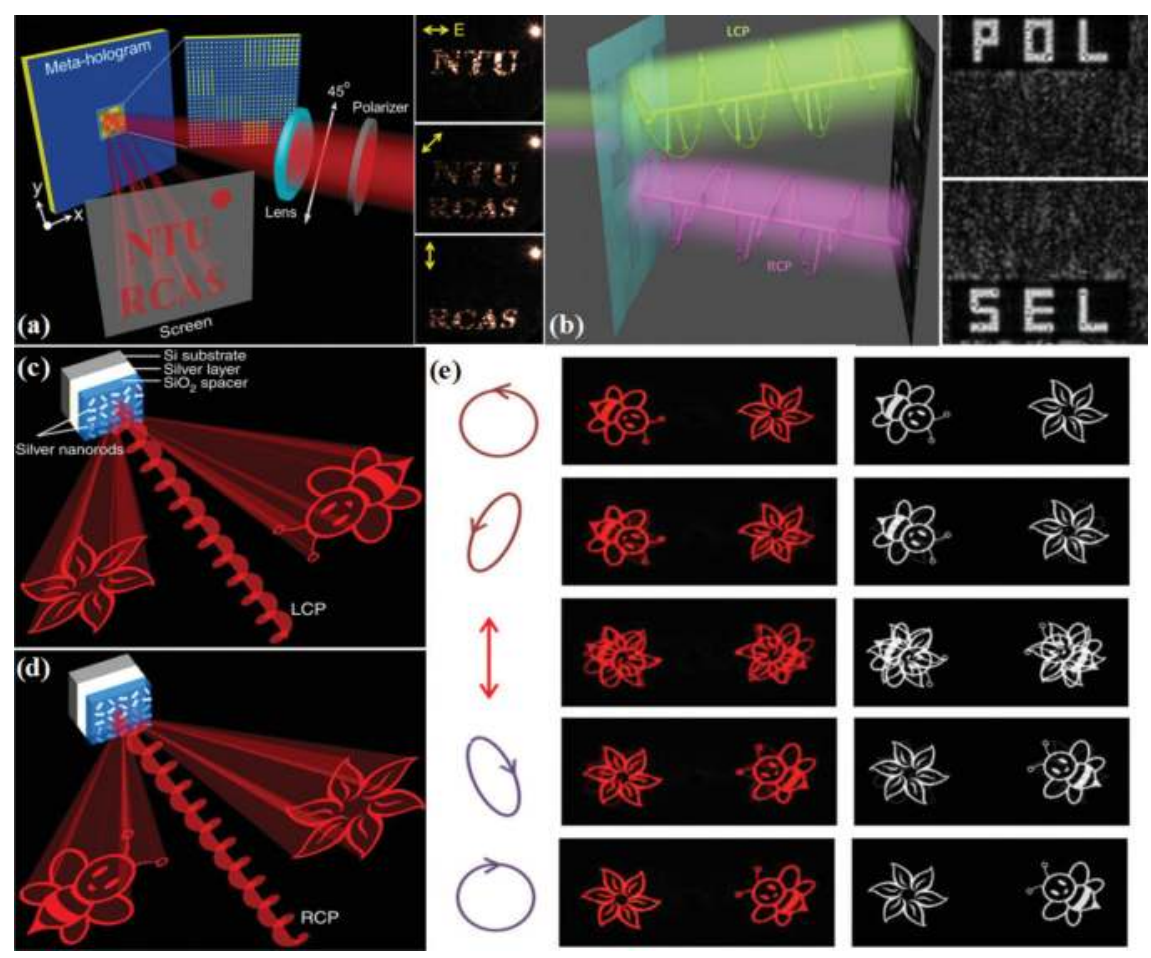

Figure 10. (a) An experimental demonstration of a polarization-switchable phase hologram and its experimental results. Reprinted by permission from [75] copyright 2013 American Chemical Society. (b) A schematic representation of polarization selective beam shaping in dielectric metamaterial device and its simulated results. Reprinted by permission from [76] copyright 2015 Optical Society of American. (c) and (d) Schematics of the helicity multiplexed hologram in nanorods based metamaterial device. (e) Reconstructed images versus incident polarization states in nanorod-based metamaterial device. The figures in the middle column and right column represent the experimental results and the corresponding simulation results, respectively. Reprinted by permission from Macmillan Publishers Ltd [77], Nature Communications copyright 2015. 
manipulate electromagnetic waves through different coding sequences of " 0 " and " 1 " elements. For example, under the periodic coding sequence of $010101 . . / 010101 \ldots$, the normally incident beam will mainly be reflected to two symmetrically oriented directions by the metasurface, whereas under the periodic coding sequence of $010101 \ldots / 101010 \ldots / 010101 \ldots / 101010$ ..., the normally incident beam will mainly be reflected to four symmetrically oriented directions, as illustrated in Figure $\mathbf{9 j}$ and $\mathbf{k}$. Based on the concept of coding metamaterials, we are able to not only control electromagnetic waves by changing the coding sequences of " 0 " and " 1 " (or " 00, , " 01, , "' 10 ," and " 11 ") elements but also create actual digital metamaterial device and programmable metamaterial device by dynamically manipulating the coding sequence. This design provides an effective way to realize the controlling of the radiation beams of antennas and reduce the scattering features of targets.

\subsubsection{Polarization-switchable phase holograms with metamaterial devices}

One of the prime goals for metamaterial research is realizing the manipulation of refractive index via using the concept of effective index and designing structures at the subwavelength scale. Metamaterials composed of subwavelength structures allow for the use of effective medium approach to describe their electromagnetic waves response in phase and amplitude sensitive to both shape and orientation of the structures. With this character, holograms which manifest itself as reconstructing predesigned images have been advanced dramatically by using metamaterial devices [71-77]. These days, polarization-switchable holograms that can separate the readout electromagnetic waves by its polarization to reconstruct different holographic images, lead to various applications such as image processing and multilevel optical switching. Figure 10a shows a plasmonic meta-hologram using metamaterial devices [75]. The meta-hologram sample consists of pixels made of $6 \times 6$ cross-nanoantennas of 16 different shapes for the phase modulation that yields polarization-controlled dual images. Figure 10b shows the demonstration of arbitrary polarization selective beam shaping with a dielectric metamaterial device [76]. Taking advantage of the conjugate phase modulation obtained by illuminating the device with LCP and RCP, two independent images, for the two orthogonal polarization states has been demonstrated. And Figure 10c-e show an experimental demonstration of helicity multiplexed metasurface hologram capable of achieving high efficiency and high image quality in the visible and near-infrared range [77]. Unlike previously demonstrated polarization multiplexed holograms that are sensitive to linear polarization, two off-axis images are interchangeable in one identical hologram by controlling the helicity of the incident wave in this work. These devices show endless possibilities for the development of holograms and provide an effective way for data storage and information processing.

\section{Challenge in future}

In previous sections, we overlooked the whole area of metamaterial-based polarization converter, and undoubtedly the achievements made by the researchers are remarkable: full control of polarization now can be accomplished with a single subwavelength film. However, there are still some challenges that stand in front of our path to the full control of electromagnetic waves. For example, how can we in depth manipulate polarization, phase, and amplitude of the incidence independently? How can we integrate different functional designs together, which are compatible with existing photonic devices on chip, such as silicon-based complementary metal-oxide-semiconductor (CMOS) photonics platform? How can we get 


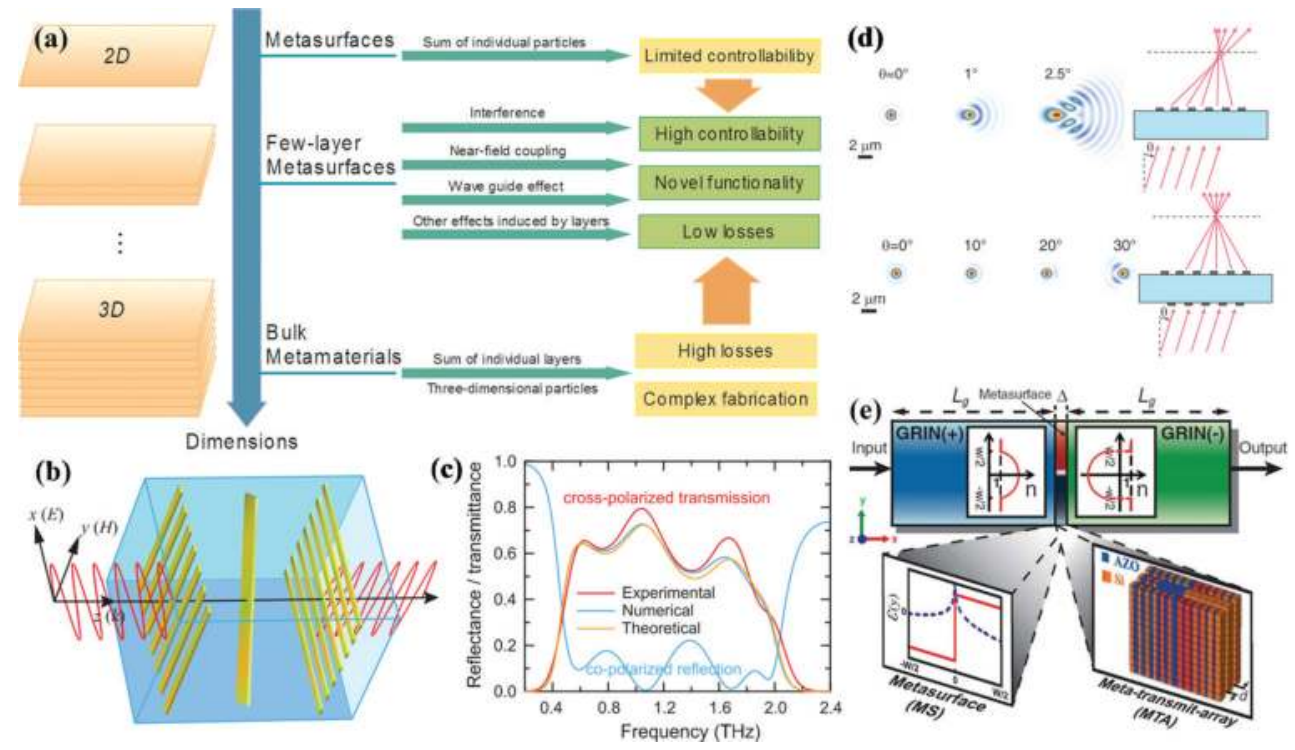

Figure 11. (a) Schematic representation of different metamaterials and their properties induced by various effects. Reprinted by permission from [25] copyright 2015 by John Wiley and Sons, Weinheim. (b) Schematic representation of the unit cell of a tri-layer metasurface linear polarization converter and (c) cross-polarized transmittance obtain through experimental measurements, numerical simulations, and theoretical calculations, together with the numerical copolarized reflection. Reprinted by permission from [81] copyright 2013 AAAS. (d) Simulated focal plane intensity for different incident angle with or without the correcting metasurface. Reprinted by permission from [82]. (e) Illustration of the system that can perform mathematical operations. A properly designed metasurface is sandwiched between two GRIN structures with positive and negative parameters. Reprinted by permission from [83] copyright 2014 AAAS.

tremendously active or tunable devices without refabricating the metamaterials? How can we achieve ultrashort response with metamaterials to obtain ultrafast detect?

One way to solving these problems may be utilizing few-layer metasurfaces [25], the principle of which is illustrated in Figure 11a. The properties of metasurfaces are sum of the individual particles (unit cells), which also means their functionalities are limited by each of the particles. For example, electric-magnetic dipoles supported by metallic nanoantennas and Mie-scattering by dielectric particles are often insufficient for independently manipulating each degree of freedom in electromagnetic waves, since each degree tends to mix up at the resonant frequency. However, other effects occur when adding another layer or layers upon the metasurfaces, such as interference [78], near-field coupling [79], waveguide effects [80], and so on, which may take advantage of each layer and even far better properties can be achieved with suitable designs. Compared with bulk metamaterials, few-layer metasurfaces are much more sufficient with easier fabrication, which are highly crucial for practical utilization. Figure 11b shows a transmitted cross-polarization converter consisting of two orthogonally positioned gratings and a low-efficiency polarization converter in the middle [81]. A Fabry-Pérot resonance occurs within the ultrathin polarization rotator, which can enhance the polarization conversion efficiency tremendously. The experimental and numerical results are shown in Figure 11c, which can be further optimized according to the authors. Few-layer metasurfaces can also be utilized as correcting layers [82]. As shown in Figure 11d, single-layer metasurfaces can easily obtain foci with hyperbolic phase distribution. However, aberration happens with oblique incidence, which is harmful as a camera 
lens. This problem can be solved by adding another correcting metasurface, and the incident angle can be increased to $30^{\circ}$, as shown in Figure 11d. Another really promising consequence achieved by few-layer metasurfaces is mathematical operation. As shown in Figure 11e, Silva et al. demonstrated a method to carry out complex mathematical operations in the Fourier domain [83]. The operation can be divided to three parts: the first and last parts involve Fourier and inverse Fourier transforms, which are realized by a graded-index (GRIN) dielectric index. Actual operations are performed by carefully designing the metasurface in the second part. If the permeability and permittivity of the metasurfaces conform with a certain relationship, differentiation, integration, and convolution can be precisely calculated. This technique requires total manipulation of the wavefront of incident electromagnetic wave with no reflection and normal wave, and this can only be realized by few-layer metasurfaces.

\section{Summary}

In this chapter, we have reviewed the recent progress in the field of polarization state manipulation of electromagnetic waves with metamaterials and discussed its wide applications in nanophotonics. Polarization state is one of the most important characteristics for electromagnetic waves, therefore arbitrary manipulation of polarization state with meta-materials provides endless possibilities for direct manipulation of electromagnetic waves and offers a powerful way for design of novel devices. With in-depth subwavelength designs, full control of polarization can now be obtained with metallic or high-contrast dielectric-based meta-materials. Furthermore, few-layer metasurfaces may provide more degree of freedom to manipulate electromagnetic waves without decrease of efficiency or increase of fabricating complexity compared to bulk metamaterials or traditional metasurfaces. With the development of metamaterial fabrication technology, meta-material devices for polarization state manipulation of electromagnetic waves are expected to have deep impact on real applications in our life.

\section{Author details}

Shuqi Chen*, Wenwei Liu, Zhancheng Li, Hua Cheng and Jianguo Tian

*Address all correspondence to: schen@nankai.edu.cn

The Key Laboratory of Weak Light Nonlinear Photonics, Ministry of Education, School of Physics and TEDA Institute of Applied Physics, Nankai University, Tianjin, China

\section{References}

[1] Zheludev N. I. The road ahead for metamaterials. Science. 2010;328(5978):582-583. DOI: 10.1126/science.1186756

[2] Zheludev N. I. Obtaining optical properties on demand. Science. 2015;348(6238):973974. DOI: $10.1126 /$ science.aac4360 
[3] Liu Y., Zhang X. Metamaterials: a new frontier of science and technology. Chemical Society Reviews. 2011;40(5):2497-2507. DOI: 10.1039/C0CS00184H

[4] Meinzer N., Barnes W. L., Hooper I. R. Plasmonic meta-atoms and metasurfaces. Nature Photonics. 2014;8(12):889-898. DOI: 10.1038/nphoton.2014.247

[5] Estakhri N. M., Alù A. Recent progress in gradient metasurfaces. JOSA B. 2016;33(2):A21A30. DOI: 10.1364/JOSAB.33.000A21

[6] Schurig D., Mock J. J., Justice B. J., Cummer S. A., Pendry J. B., Starr A. F., et al. Metamaterial electromagnetic cloak at microwave frequencies. Science. 2006;314(5801):977-980. DOI: $10.1126 /$ science. 1133628

[7] Pendry J. B., Schurig D., Smith D. R. Controlling electromagnetic fields. Science. 2006;312(5781):1780-1782. DOI: 10.1126/science.1125907

[8] Ni X., Wong Z. J., Mrejen M., Wang Y., Zhang X. An ultrathin invisibility skin cloak for visible light. Science. 2015;349(6254):1310-1314. DOI: 10.1126/science.aac9411

[9] Kildishev A. V., Boltasseva A., Shalaev V. M. Planar photonics with metasurfaces. Science. 2013;339(6125):1232009. DOI: 10.1126/science.1232009

[10] Yu P., Chen S., Li J., Cheng H., Li Z., Liu W., et al. Dynamically tunable plasmonic lens between the near and far fields based on composite nanorings illuminated with radially polarized light. Plasmonics. 2015;10(3):625-631. DOI: 10.1007/s11468-014-9848-3

[11] Shelby R. A., Smith D. R., Schultz, S. Experimental verification of a negative index of refraction. Science. 2001;292(5514):77-79. DOI: 10.1126/science.1058847

[12] Khorasaninejad M., Chen W. T., Devlin R. C., Oh J., Zhu A. Y., Capasso, F. Metalenses at visible wavelengths: diffraction-limited focusing and subwavelength resolution imaging. Science. 2016;352(6290):1190-1194. DOI: 10.1126/science.aaf6644

[13] Liu Z., Li Z., Liu Z., Li J., Cheng H., Yu, P., et al. High-performance broadband circularly polarized beam deflector by mirror effect of multinanorod metasurfaces. Advanced Functional Materials. 2015;25(34):5428-5434. DOI: 10.1002/adfm.201502046

[14] Li J., Chen S., Yang H., Li J., Yu P., Cheng H., et al. Simultaneous control of light polarization and phase distributions using plasmonic metasurfaces. Advanced Functional Materials. 2015;25(5):704-710. DOI: 10.1002/adfm.201403669

[15] Litchinitser, N. M. Photonic multitasking enabled with geometric phase. Science. 2016;352(6290):1177-1178. DOI: 10.1126/science.aaf8391

[16] Zhu A. Y., Kuznetsov A. I., Luk'yanchuk B., Engheta N., Genevet P. Traditional and emerging materials for optical metasurfaces. Nanophotonics. 2016; DOI: 10.1515/ nanoph-2016-0032

[17] Yu N., Capasso F. Flat optics with designer metasurfaces. Nature materials. 2014;13(2):139-150. DOI: 10.1038/nmat3839

[18] Saleh B. E., Teich M. C., Masters B. R. Fundamentals of photonics, Second Edition. Journal of Biomedical Optics. 2008;13(4):9901. DOI: 10.1117/1.2976006 
[19] Gordon R., Brolo A. G., McKinnon A., Rajora A., Leathem B., Kavanagh K. L. Strong polarization in the optical transmission through elliptical nanohole arrays. Physical Review Letters. 2004;92(3):037401. DOI: 10.1103/PhysRevLett.92.037401

[20] Sung J., Sukharev M., Hicks E. M., Van Duyne R. P., Seideman T., Spears K. G. Nanoparticle spectroscopy: birefringence in two-dimensional arrays of L-shaped silver nanoparticles. The Journal of Physical Chemistry C. 2008;112(9):3252-3260. DOI: 10.1021/jp077389y

[21] Li T., Liu H., Wang S. M., Yin X. G., Wang F. M., Zhu S. N., et al. Manipulating optical rotation in extraordinary transmission by hybrid plasmonic excitations. Applied Physics Letters. 2008;93(2):021110. DOI: 10.1063/1.2958214

[22] Ellenbogen T., Seo K., Crozier K. B. Chromatic plasmonic polarizers for active visible color filtering and polarimetry. Nano Letters. 2012;12(2):1026-1031. DOI: 10:1021/ $\mathrm{nl} 204257 \mathrm{~g}$

[23] Zhao Y., Alu`A. Tailoring the dispersion of plasmonic nanorods to realize broadband optical meta-waveplates. Nano Letters. 2013;13(3):1086-1091. DOI: 10.1021/nl304392b

[24] Pors A., Nielsen M. G., Della Valle G., Willatzen M., Albrektsen O., Bozhevolnyi S. I. Plasmonic metamaterial wave retarders in reflection by orthogonally oriented detuned electrical dipoles. Optics Letters. 2011;36(9):1626-1628. DOI: 10.1364/OL.36.001626

[25] Cheng H., Liu Z., Chen S., Tian J. Emergent functionality and controllability in few-layer metasurfaces. Advanced Materials. 2015;27(36):5410-5421. DOI: 10.1002/ adma.201501506

[26] Jahani S., Jacob Z. All-dielectric metamaterials. Nature Nanotechnology. 2016;11(1):2326. DOI: $10.1038 /$ nnano.2015.304

[27] Lévesque Q., Makhsiyan M., Bouchon P., Pardo F., Jaeck J., Bardou N. Plasmonic planar antenna for wideband and efficient linear polarization conversion. Applied Physics Letters. 2014;104(11):111105. DOI: 10.1063/1.4869127

[28] Jiang Z. H., Lin L., Ma D., Yun S., Werner D. H., Liu Z., et al. Broadband and wide fieldof-view plasmonic metasurface-enabled waveplates. Scientific Reports. 2014;4:7511. DOI: $10.1038 /$ srep07511

[29] Li T., Wang S. M., Cao J. X., Liu H., Zhu S. N. Cavity-involved plasmonic metamaterial for optical polarization conversion. Applied Physics Letters. 2010;97(26):261113. DOI: $10.1063 / 1.3533912$

[30] Li Z., Chen S., Liu W., Cheng H., Liu Z., Li J, et al. High performance broadband asymmetric polarization conversion due to polarization-dependent reflection. Plasmonics. 2015;10(6):1703-1711. DOI: 10.1007/s11468-015-9986-2

[31] Li Z., Zheng G., He P. A., Li S., Deng Q., Zhao J., et al. All-silicon nanorod-based Dammann gratings. Optics Letters. 2015;40(18):4285-4288. DOI: 10.1364/OL.40.004285

[32] Wu C., Arju N., Kelp G., Fan J. A., Dominguez J., Gonzales E. Spectrally selective chiral silicon metasurfaces based on infrared Fano resonances. Nature Communications. 2014;5:3892. DOI: 10.1038/ncomms4892 
[33] Stalder M., Schadt M. Linearly polarized light with axial symmetry generated by liquidcrystal. Optics Letters. 1996;21(23): 1948-1950. DOI: 10.1364/OL.21.001948

[34] Prati E. Propagation in gyroelectromagnetic guiding systems. Journal of Electromagnetic Waves and Applications. 2003;17(8): 1177-1196. DOI: 10.1163/156939303322519810

[35] Arbabi A., Horie Y., Bagheri M., Faraon A. Dielectric metasurfaces for complete control of phase and polarization with subwavelength spatial resolution and high transmission. Nature Nanotechnology. 2015;10:937-943. DOI: 10.1038/NNANO.2015.186

[36] Wu S., Zhang Z., Zhang Y., Zhang K., Zhou L., Zhang X., et al. Enhanced rotation of the polarization of a light beam transmitted through a silver film with an array of perforated S-shaped holes. Physical Review Letters. 2013;110(20): 207401. DOI: 10.1103/ PhysRevLett.110.207401

[37] Liu W., Chen S., Li Z., Cheng H., Yu P., Li J., et al. Realization of broadband crosspolarization conversion in transmission mode in the terahertz region using a single-layer metasurface. Optics Letters. 2015;40(13):3185-3188. DOI: 10.1364/OL.40.003185

[38] Yu P., Li J., Tang C., Cheng H., Liu Z., Li Z., et al. Controllable optical activity with nonchiral plasmonic metasurfaces. Light: Science and Applications. 2016;5(7):e16096. DOI: 10.1038/lsa.2016.96

[39] Li Z., Liu W., Cheng H., Chen S., Tian J. Realizing broadband and invertible linear-to-circular polarization converter with ultrathin single-layer metasurface. Scientific Reports. 2015;5:18106. DOI: 10.1038/srep18106

[40] Yin X., Ye Z., Rho J., Wang Y., Zhang X. Photonic spin Hall effect at metasurfaces. Science. 2013;339(6126): 1405-1407. DOI: 10.1126/science.1231758

[41] Yang Y., Wang W., Moitra P., Kravchenko I. I., Briggs D. P., Valentine, J. Dielectric metareflect array for broadband linear polarization conversion and optical vortex generation. Nano Letters. 2014;14(3): 1394-1399. DOI: 10.1021/nl4044482

[42] Li L., Li T., Tang X. M., Wang S. M., Wang Q. J., Zhu S. N. Plasmonic polarization generator in well-routed beaming. Light: Science and Applications. 2015;4(9):e330. DOI: 10.1038/1sa.2015.103

[43] Kruk S., Hopkins B., Kravchenko I., Miroshnichenko A., Neshev D. N., Kivshar Y. S. Broadband highly-efficient dielectric metadevices for polarization control. APL Photonics. 2016; 1(3). DOI: 10.1063/1.4949007

[44] Menzel C., Helgert C., Rockstuhl C., Kley E. B., Tünnermann A., Pertsch T., et al. Asymmetric transmission of linearly polarized light at optical metamaterials. Physical Review Letters. 2010;104(25):253902. DOI: 10.1103/PhysRevLett.104.253902

[45] Pfeiffer C., Grbic A. Bianisotropic metasurfaces for optimal polarization control: analysis and synthesis. Physical Review Applied. 2014;2(4):044011. DOI: 10.1103/ PhysRevApplied.2.044011

[46] Xu T., Lezec H. J. Visible-frequency asymmetric transmission devices incorporating a hyperbolic metamaterial. Nature Communications. 2014;5:4141. DOI: 10.1038/ ncomms5141 
[47] Li Z., Chen S., Tang C., Liu W., Cheng H., Liu Z., et al. Broadband diodelike asymmetric transmission of linearly polarized light in ultrathin hybrid metamaterial. Applied Physics Letters. 2014;105(20):201103. DOI: 10.1063/1.4902162

[48] Ye Y., He S. $90^{\circ}$ polarization rotator using a bilayered chiral metamaterial with giant optical activity. Applied Physics Letters. 2010;96(20):203501. DOI: 10.1063/1.3429683

[49] Han J., Li H., Fan Y., Wei Z., Wu C., Cao Y., et al. An ultrathin twist-structure polarization transformer based on fish-scale metallic wires. Applied Physics Letters. 2011;98(15):151908. DOI: 10.1063/1.3580608

[50] Khatua S., Chang W. S., Swanglap P., Olson J., Link, S. Active modulation of nanorod plasmons. Nano Letters. 2011;11(9):3797-3802. DOI: 10.1021/nl201876r

[51] Shen N. H., Massaouti M., Gokkavas M., Manceau J. M., Ozbay E., Kafesaki M., et al. Optically implemented broadband blueshift switch in the terahertz regime. Physical Review Letters. 2011;106(3):037403. DOI: 10.1103/PhysRevLett.106.037403

[52] Zharov A. A., Shadrivov I. V., Kivshar Y. S. Nonlinear properties of left-handed metamaterials. Physical Review Letters. 2003;91(3):037401. DOI: 10.1103/ PhysRevLett.91.037401

[53] Shaltout A. M., Kildishev A. V., Shalaev V. M. Evolution of photonic metasurfaces: from static to dynamic. JOSA B. 2016;33(3):501-510. DOI: 10.1364/JOSAB.33.000501

[54] Zheludev N. I., Plum E. Reconfigurable nanomechanical photonic metamaterials. Nature Nanotechnology. 2016;11(1):16-22. DOI: 10.1038/nnano.2015.302

[55] Novoselov K. S., Geim A. K., Morozov S. V., Jiang D., Zhang Y., Dubonos S. V., et al. Electric field effect in atomically thin carbon films. Science. 2004;306(5696):666-669. DOI: 10.1126/science.1102896

[56] Emani N. K., Chung T. F., Ni X., Kildishev A. V., Chen Y. P., Boltasseva A. Electrically tunable damping of plasmonic resonances with graphene. Nano Letters. 2012;12(10): 5202-5206. DOI: 10.1021/nl302322t

[57] Valmorra F., Scalari G., Maissen C., Fu W., Schönenberger C., Choi J. W., et al. Low-bias active control of terahertz waves by coupling large-area CVD graphene to a terahertz metamaterial. Nano Letters. 2013;13(7):3193-3198. DOI: 10.1021/nl4012547

[58] Cheng H., Chen S., Yu P., Li J., Deng L., Tian J. Mid-infrared tunable optical polarization converter composed of asymmetric graphene nanocrosses. Optics Letters. 2013;38(9):1567-1569. DOI: 10.1364/OL.38.001567

[59] Cheng H., Chen S., Yu P., Li J., Xie B., Li Z., et al. Dynamically tunable broadband midinfrared cross polarization converter based on graphene metamaterial. Applied Physics Letters. 2013;103(22):223102. DOI: 10.1063/1.4833757

[60] Cheng H., Chen S., Yu P., Liu W., Li Z., Li J., et al. Dynamically tunable broadband infrared anomalous refraction based on graphene metasurfaces. Advanced Optical Materials. 015;3(12):1744-1749. DOI: 10.1002/adom.201500285

[61] Bliokh K. Y., Rodríguez-Fortuño F. J., Nori F., Zayats A. V. Spin-orbit interactions of light. Nature Photonics. 2015;9(12):796-808. DOI: 10.1038/NPHOTON.2010.201 
[62] Ling X., Zhou X., Shu W., Luo H., Wen S. Realization of tunable photonic spin Hall effect by tailoring the Pancharatnam-Berry phase. Scientific Reports. 2014;4:5557. DOI: 10.1038/srep05557

[63] Shu W., Ke Y., Liu Y., Ling X., Luo H., Yin X. Radial spin Hall effect of light. Physical Review A. 2016;93(1):013839. DOI: 10.1103/PhysRevA.93.013839

[64] Liu Y., Ling X., Yi X., Zhou X., Chen S., Ke Y., et al. Photonic spin Hall effect in dielectric metasurfaces with rotational symmetry breaking. Optics Letters. 2015;40(5):756-759. DOI: 10.1364/OL.40.000756

[65] Ling X., Zhou X., Yi X., Shu W., Liu Y., Chen S., et al. Giant photonic spin Hall effect in momentum space in a structured metamaterial with spatially varying birefringence. Light: Science and Applications. 2015;4(5):e290. DOI: 10.1038/lsa.2015.63

[66] Li J., Yu P., Cheng H., Liu W., Li Z., Xie B., et al. Optical polarization encoding using graphene-loaded plasmonic metasurfaces. Advanced Optical Materials. 2016;4(1):91-98. DOI: 10.1002/adom.201500398

[67] Cui T. J., Qi M. Q., Wan X., Zhao J., Cheng Q. Coding metamaterials, digital metamaterials and programmable metamaterials. Light: Science and Applications. 2014;3(10):e218. DOI: $10.1038 /$ lsa.2014.99

[68] Gao L. H., Cheng Q., Yang J., Ma S. J., Zhao J., Liu S., et al. Broadband diffusion of terahertz waves by multi-bit coding metasurfaces. Light: Science and Applications. 2015;4(9):e324. DOI: doi:10.1038/lsa.2015.97

[69] Liu S., Cui T. J., Xu Q., Bao D., Du L., Wan X., et al. Anisotropic coding metamaterials and their powerful manipulation of differently polarized terahertz waves. Light: Science and Applications. 2016;5(5):e16076. DOI: 10.1038/lsa.2016.76

[70] Wang K., Zhao J., Cheng Q., Cui T. J. Broadband and broad-angle low-scattering metasurface based on hybrid optimization algorithm. Scientific Reports. 2014;4:5935. DOI: 10.1038/srep05935

[71] Zheng G., Mühlenbernd H., Kenney M., Li G., Zentgraf T., Zhang, S. Metasurface holograms reaching 80\% efficiency. Nature Nanotechnology. 2015;10(4):308-312. DOI: 10.1038/NNANO.2015.2

[72] Yifat Y., Eitan M., Iluz Z., Hanein Y., Boag A., Scheuer J. Highly efficient and broadband wide-angle holography using patch-dipole nanoantenna reflectarrays. Nano Letters. 2014;14(5):2485-2490. DOI: 10.1021/n15001696

[73] Ni X., Kildishev A. V., Shalaev V. M. Metasurface holograms for visible light. Nature Communications. 2013;4:2807. DOI: 10.1038/ncomms3807

[74] Khorasaninejad M., Ambrosio A., Kanhaiya P., Capasso, F. Broadband and chiral binary dielectric meta-holograms. Science Advances. 2016;2(5):e1501258. DOI: 10.1126/ sciadv.1501258

[75] Chen W. T., Yang K. Y., Wang C. M., Huang Y. W., Sun G., Chiang I. D., et al. Highefficiency broadband meta-hologram with polarization-controlled dual images. Nano Letters. 2013;14(1):225-230. DOI: 10.1021/nl403811d 
[76] Desiatov B., Mazurski N., Fainman Y., Levy U. Polarization selective beam shaping using nanoscale dielectric metasurfaces. Optics Express. 2015;23(17):22611-22618. DOI: 10.1364/OE.23.022611

[77] Wen D., Yue F., Li G., Zheng G., Chan K., Chen S., et al. Helicity multiplexed broadband metasurface holograms. Nature Communications. 2015;6:8241. DOI: 10.1038/ ncomms9241

[78] Chen H. T. Interference theory of metamaterial perfect absorbers. Optics Express. 2012;20(7):7165-7172. DOI: 10.1364/OE.20.007165

[79] Lee J., Tymchenko M., Argyropoulos C., Chen P. Y., Lu F., Demmerle F., et al . Giant nonlinear response from plasmonic metasurfaces coupled to intersubband transitions. Nature. 2014;511(7507):65-69. DOI: 10.1038/nature13455

[80] Holloway C. L., Kuester E. F., Gordon J. A., O’Hara J., Booth J., Smith, D. R. An overview of the theory and applications of metasurfaces: the two-dimensional equivalents of metamaterials. IEEE Antennas and Propagation Magazine. 2012;54(2):10-35. DOI: 10.1109/MAP.2012.6230714

[81] Grady N. K., Heyes J. E., Chowdhury D. R., Zeng Y., Reiten M. T., Azad A. K., et al. Terahertz metamaterials for linear polarization conversion and anomalous refraction. Science. 2013;340(6138):1304-1307. DOI: 10.1126/science.1235399

[82] Arbabi A., Arbabi E., Kamali S. M., Horie Y., Han S., and Faraon, A. Miniature optical planar camera based on a wide-angle metasurface doublet corrected for monochromatic aberrations. arXiv. Forthcoming. DOI: arXiv:1604.06160

[83] Silva A., Monticone F., Castaldi G., Galdi V., Alù A., Engheta N. Performing mathematical operations with metamaterials. Science. 2014;343(6167):160-163. DOI: 10.1126/ cience.1242818 